\title{
WEAKLY REDUCTIVE SEMIGROUPS WITH ATOMISTIC CONGRUENCE LATTICES
}

\author{
KARL AUINGER \\ (Received 30 January 1989; revised 17 May 1989)
}

Communicated by P. G. Trotter

\begin{abstract}
The structure of semigroups with atomistic congruence lattices (that is, each congruence is the supremum of the atoms it contains) is studied. For the weakly reductive case the problem of describing the structure of such semigroups is solved up to simple and congruence free semigroups, respectively. As applications, all commutative, finite, completely semisimple semigroups, respectively, with atomistic congruence lattices are described.
\end{abstract}

1980 Mathematics subject classification (Amer. Math. Soc.) (1985 Revision): 20 M 10, 08 A 30.

\section{Introduction and Preliminaries}

A lattice is atomistic if each element is the supremum of the atoms it contains. Examples are the chain of two elements, the power set lattice of a set or the partition lattice of some set. In [3] it is shown that a semilattice has an atomistic congruence lattice if and only if it is a locally finite tree. In this paper we study the structure of semigroups whose congruence lattices are atomistic. Examples are congruence free semigroups (as a trivial case), left (right) zero semigroups, null semigroups, rectangular bands and semigroups whose congruence lattice is Boolean. In the second section we obtain necessary conditions on a semigroup in order that its congruence lattice be atomistic. The main tool for investigating the structure of such semigroups $S$ is to consider the decomposition of $S$ into its $J$-classes. We introduce

(C) 1990 Australian Mathematical Society $0263-6115 / 90 \$ A 2.00+0.00$ 
the construction of trees of 0-simple semigroups and show that each globally idempotent semigroup with an atomistic congruence lattice can be so constructed (Theorem 1). The structure of such a semigroup can be described by a locally finite tree $X, 0$-simple semigroups $I_{\alpha}$ indexed by the elements of $X$ and partial homomorphisms between the nonzero parts of the semigroups $I_{\alpha}$. Furthermore, if an arbitrary semigroup has an atomistic congruence lattice then it is an inflation of such a semigroup.

In Section 3 we study the problem for weakly reductive semigroups and obtain necessary and sufficient conditions in order that the congruence lattice be atomistic (Theorem 2). Using this characterization we are able to characterize all commutative, finite and completely semisimple semigroups with atomistic congruence lattices (up to locally finite trees and simple groups). This will be done in Section 4. Furthermore, we observe that the properties "atomistic" and "Boolean", "complemented modular" and "relatively complemented" for the congruence lattice of a weakly reductive semigroup are strongly connected.

For the remainder of this part we collect some definitions and results which are basic for our considerations (for further details see [4] or [6]).

A semilattice is a (locally finite) tree if each interval $[x, y]=\{z: x \leq z \leq$ $y\}$ is a (finite) chain. For a semigroup $S, S^{*}=S$ if $S$ has no zero and $S^{*}=$ $S \backslash\{0\}$ if 0 is the zero of $S$; and $S^{1}=S$ if $S$ has an identity and $S^{1}=S \cup\{1\}$ such that $1 \notin S$ and $s 1=1 s=s$ for all $s \in S$ otherwise. Green's relation $J$ is defined by a $J b$ if and only if $J(a)=J(b)$, where $J(x)$ is the principal ideal generated by $x$ (that is, $J(x)=S^{1} x S^{1}$ ). The J-class containing $a$ is denoted by $J_{a}$. The set $I(a)=J(a) \backslash J_{a}=\{x \in J(a): J(x) \neq J(a)\}$ is an ideal in $J(a)$ (or empty). The semigroup $J(a) / I(a)$ is called a principal factor. Each principal factor is either simple, 0 -simple or null (see [4]). A semigroup is (completely) semisimple if each principal factor is (completely) (0)-simple. Let $S$ be a subsemigroup of a semigroup $T$. Then $T$ is an inflation of $S$ if there exists a function $f: T \rightarrow S$ such that $f \mid S=\mathrm{id}_{S}$ and $a b=(a f)(b f)$ for all $a, b \in T$. In this case $f$ is the inflation function. A semigroup $S$ is weakly reductive if for $a, b \in S, z a=z b$ and $a z=b z$ for all $z \in S$ imply $a=b$. A semigroup $S$ is globally idempotent if $S^{2}=S$. The lattice of all congruences on a semigroup $S$ is denoted by Con $S$. The identical and the universal relations are denoted by $\varepsilon_{S}=\varepsilon$ and $\omega_{S}=\omega$, respectively. A congruence $\rho$ on $S$ is an atom if it covers $\varepsilon$, to be denoted by $\rho \succ \varepsilon$, that is, $\varepsilon<\rho$ and $[\varepsilon, \rho]=\{\varepsilon, \rho\}$. The set of all atoms of Con $S$ is denoted by At $S$. For an arbitrary relation $R$ on $S, R^{*}$ is the congruence on $S$ which is generated by $R$.

For 0 -simple semigroups we have the following result. 
Result 1 [5]. A 0-simple semigroup is congruence free if and only if for any two distinct elements $x, y \in S$ there exist $u, v \in S$ such that $u x v=0$ and $u y v \neq 0$ or $u x v \neq 0$ and $u y v=0$.

\section{Trees of 0 -simple semigroups}

In this section we obtain necessary conditions on an arbitrary semigroup $S$ in order that its congruence lattice be atomistic. For this purpose we study the decomposition of $S$ into its J-classes and the ordered set $S / J$. We introduce the construction of trees of 0 -simple semigroups and show that each globally idempotent semigroup $S$ whose congruence lattice is atomistic can be so constructed.

Lemma 1. Let $\rho \in$ At $S$ and $a \rho b$ for $J_{a}>J_{b}$. Then $J_{x}<J_{a}$ implies that $J_{x} \leq J_{b}$. In this case $J(a)=J_{a} \cup J(b)$.

Proof. Let $x=s a t$ for $s, t \in S^{1}$. Then $x=s a t \rho s b$. Since $J_{x}$ and $J_{b}$ are contained in $I(a)$ we obtain that $s a t=s b t$. Otherwise $(s a t, s b t)^{*}$ is a proper congruence which is strictly contained in $\rho$.

Conversely, if Con $S$ is atomistic any two of such neighbours in $S / J$ are "linked" by an atom.

LEMMA 2. Let $J_{b}<J_{a}$ and assume that $J_{x}<J_{a}$ implies that $J_{x} \leq J_{b}$. If $\operatorname{Con} S$ is atomistic then there exists $v \in J_{b}$ and an atom $\rho$ such that $a \rho v$.

Proof. We consider the congruence $(a, b)^{*}$. Since Con $S$ is atomistic there exist $\rho_{1}, \ldots, \rho_{n} \in$ At $S$ such that $a=a_{0} \rho_{1} a_{1} \cdots \rho_{n} a_{n}=b$ for certain $a_{i} \in S$ and $\rho_{i} \subseteq(a, b)^{*}$. Since $\{a, b\} \subseteq J(a)$, all $a_{i}$ are contained in $J(a)$, that is, $J_{a_{i}} \leq J_{a}$. Let $i$ be the smallest index such that $J_{a_{i}}<J_{a_{i-1}}=J_{a}$. The assumption on $J_{b}$ then implies that $J_{a_{i}} \leq J_{b}$. Since $J_{a}=J_{a_{i-1}}$ and $a_{i-1} \rho_{i} a_{i}$, by Lemma 1 , we get that $J_{a_{i}} \geq J_{b}$ and thus $J_{a_{i}}=J_{b}$. Also $a J a_{i-1}$ implies that $a=s a_{i-1} t$ for some $s, t \in S^{1}$ and thus $a=s a_{i-1} t \rho_{i} s a_{i} t$. Now $J_{s a_{i} t} \leq J_{b}$ and Lemma 1 imply that $J_{s a_{i} t}=J_{b}$.

Lemma 3. If Con $S$ is atomistic then $S / J$ is a locally finite tree.

Proof. $S / J$ is directed so it suffices to show that each interval in $S / J$ is a finite chain. Let $J_{a}>J_{b}$; there exist atoms $\rho_{1}, \ldots, \rho_{n}$ such that $a=b_{0} \rho_{1} b_{1} \cdots \rho_{n} b_{n}=b$ and $b_{i} \in J(a)$ for all $i$. Let $g(a, b)=n$ be the shortest possible length of all such sequences and 


$$
h\left(J_{a}, J_{b}\right)=\min \{g(x, y): x J a, y J b\} .
$$

By induction on $h\left(J_{a}, J_{b}\right)$ we show that each interval in $S / J$ is a finite chain. If $h\left(J_{a}, J_{b}\right)=1$ then an immediate consequence of Lemma 1 is that $\left[J_{b}, J_{a}\right]=\left\{J_{b}, J_{a}\right\}$. Let $J_{b}<J_{a}, h\left(J_{a}, J_{b}\right)=n>1$ and suppose that $\left[J_{x}, J_{y}\right]$ is a finite chain whenever $h\left(J_{y}, J_{x}\right)<n$. Let $u=a_{0} \rho_{1} a_{1} \cdots \rho_{n} a_{n}=$ $v$ for certain $\rho_{i} \in \operatorname{At} S$ such that $u J a, v J b$ and $a_{i} \in J(a)$ for all $i$. In particular, $J_{a_{i}} \leq J_{a}$. If $J_{a_{i}}=J_{a}$ then $h\left(J_{a}, J_{b}\right)=h\left(J_{a_{i}}, J_{b}\right)<n$, a contradiction. Therefore $J_{a_{i}}<J_{a}$. Also, since $J(a)=J_{a} \cup J\left(a_{1}\right)$ then $a_{i} \in J\left(a_{1}\right)$. Therefore, $J_{a_{1}}<J_{a}$ and $a_{k} \in J\left(a_{1}\right)$ for all $k \geq 1$. In particular $h\left(J_{a_{1}}, J_{b}\right)<n$ and our assumption applies: $\left[J_{b}, J_{a_{1}}\right]$ is a finite chain. If $J_{x} \in\left[J_{b}, J_{a}\right]$ then $J_{x}=J_{a}$ or $J_{x} \leq J_{a_{1}}$. Therefore $\left[J_{b}, J_{a}\right]=\left[J_{b}, J_{a_{1}}\right] \cup$ $\left\{J_{a}\right\}$ is a finite chain.

In the next statements let us assume that $\operatorname{Con} S$ is atomistic.

LEMMA 4. Let $J_{a} \succ J_{b}$. Then there exists a partial homomorphism $f: J_{a} \rightarrow$ $J_{b}$ so that $x y=(x f) y$ and $y x=y(x f)$ for all $x \in J_{a}$ and $y \in S$ such that $x y, y x \in J(b)$, respectively. In particular, if $x y \in J(b)$ for $x, y \in J_{a}$ then $x y=(x f)(y f)$.

Proof. By Lemma 2, there exists an atom $\rho$ such that $a \rho u$ for some $u \in J_{b}$. Let $x \in J_{a} ; x=s a t$ for some $s, t \in S^{1}$. Then $x=$ sat $\rho$ sut . By Lemma 1 , sut $\in J_{b}$. If $x \rho v$ for some $v \in J_{b}$ then $v=$ sut since $\rho \mid J(b)=\varepsilon$. Thus for each $x \in J_{a}$ there exists a unique element in $J_{b}$ to be denoted by $x f$ such that $x \rho x f$. Let $y \in S$ such that $x y \in J(b)$. Then $x y \rho(x f) y$. Then $\rho \mid J(b)=\varepsilon$ implies that $x y=(x f) y$. Now let $x, y \in J_{a}$ such that $x y \in J_{a}$. Then $x \rho x f, y \rho y f$ and the definition of $f$ imply that $(x y) f \rho x y$ and $x y \rho(x f)(y f)$. Since $\rho \mid J(b)=\varepsilon$ we get that $f$ is a partial homomorphism.

This result can be extended to any comparable $J$-classes.

LEMMA 5. Let $J_{a}>J_{b}$. Then there exists a partial homomorphism $f: J_{a} \rightarrow$ $J_{b}$ such that $x z=(x f) z$ or $z x=z(x f)$ for all $x \in J_{a}, z \in S$ such that $x z$ or $z x \in J(b)$, respectively.

Proof. The interval $\left[J_{b}, J_{a}\right]$ is a finite chain so there exist unique $J_{a_{i}}$ such that $J_{a}=J_{a_{0}} \succ J_{a_{1}} \succ \cdots \succ J_{a_{n}} \succ J_{b}$. Let $f_{i}: J_{a_{i-1}} \rightarrow J_{a_{i}}$ be the mapping considered in Lemma 4. Let $f=f_{1} f_{2} \cdots f_{n}$. Then $f$ is a partial homomorphism and for $x \in J_{a}, z \in S$ such that $x z \in J(b)$, by Lemma 4 we obtain that $x z=\left(x f_{1}\right) z=\left(x f_{1} f_{2}\right) z=\cdots=\left(x f_{1} f_{2} \cdots f_{n}\right) z=(x f) z$. The analogous argument for $z x$ completes the proof.

Proposition 1. Each non-maximal J-class of $S$ is the non-zero part of a 0 -simple semigroup. In particular, $S^{2}$ is semisimple. 
PRoof. Let $J_{b}<J_{a} ; b=$ sat for some $s, t \in S^{1}$. Since $J_{b} \leq J_{s}, J_{a}, J_{t}$ we obtain that $b=(s f)(a g)(t h)$ where $f, g, h$ denote the mappings constructed in Lemma 5 so that $s f, a g, t h \in J_{b}$.

LEMMA 6. The semigroup $S$ is an inflation of $S^{2}$.

Proof. The case where $S=S^{2}$ is trivial. Let $a \in S \backslash S^{2}$ and let $J_{b}$ denote the unique $J$-class which is covered by $J_{a}$. Let $f_{a}: J_{a} \rightarrow J_{b}$ be the partial homomorphism constructed in Lemma 4. Let $z \in S$; $a$ cannot be written as a product so $J_{a z}<J_{a}$ and thus $J_{a z} \leq J_{b}$. By Lemma 4, $a z=\left(a f_{a}\right) z$ and by analogy, $z a=z\left(a f_{a}\right)$. Now define $f: S \rightarrow S^{2}$ by $x f=x f_{x}$ if $x \in S \backslash S^{2}$ and $x f=x$ otherwise. Then $f$ is an inflation function.

Since inflations are trivial from an algebraic point of view we consider the semisimple semigroup $S^{2}$ rather than $S$ itself. The results so far motivate the following construction.

Construction. Let $X$ be a locally finite tree, to each $\alpha \in X$ associate a 0 -simple semigroup $I_{\alpha}(\neq\{0\})$ so that $I_{\alpha} \cap I_{\beta}=\varnothing$ if $\alpha \neq \beta$. For $\alpha \in X^{*}$ let $f_{\alpha}: I_{\alpha}^{*} \rightarrow I_{\alpha^{+}}^{*}$ be a partial homomorphism where $\alpha^{+}$denotes the unique element of $X$ such that $\alpha \succ \alpha^{+}$. Let $f_{\alpha, \alpha}=\mathrm{id}_{I_{\alpha}^{*}}$ and $f_{\alpha, \beta}$ be defined by $f_{\alpha, \beta}=f_{\alpha_{1}} f_{\alpha_{2}} \cdots f_{\alpha_{n}}$ where the $\alpha_{i}$ 's are defined by $\alpha=\alpha_{1} \succ \alpha_{2} \cdots \alpha_{n} \succ \beta$. We suppose that for arbitrary $a \in I_{\alpha}^{*}$ and $b \in I_{\beta}^{*}$ the set

$$
D(a, b)=\left\{\gamma \in X:\left(a f_{\alpha, \gamma}\right)\left(b f_{\beta, \gamma}\right) \text { is defined in } I_{\gamma}^{*}\right\}
$$

is not empty. Let $\delta(a, b)$ denote the greatest element of $D(a, b)$. Let $S=\bigcup\left(I_{\alpha}^{*}: \alpha \in X\right)$ and define a multiplication $*$ on $S$ by the rule

$$
a * b=\left(a f_{\alpha, \delta(a, b)}\right)\left(b f_{\beta, \delta(a, b)}\right) \quad\left(a \in I_{\alpha}^{*}, \beta \in I_{\beta}^{*}\right)
$$

where the right hand side product is defined in $I_{\delta(a, b)}^{*}$.

Definition. The groupoid $S$ is a tree of 0-simple semigroups, to be denoted by $S=\left(X ; I_{\alpha}, f_{\alpha, \beta}\right)$. If each $I_{\alpha}, \alpha \in X$, is congruence free (with zero and not the null semigroup of order two) then $S$ is a tree of congruence free semigroups.

If $X$ has a least element $\mu$ then by definition $I_{\mu}^{*}$ is closed under multiplication and thus is a simple semigroup. If, in addition, $S$ is a tree of congruence free semigroups then the congruence freeness of $I_{\mu}^{*} \cup\{0\}$ implies that $I_{\mu}^{*}$ consists of exactly one element. A straightforward verification shows that $S$ is a semigroup. Similar constructions appear in [1], [2], [7], [9], [12].

We now are able to formulate

THeOREM 1. If $S$ is globally idempotent and $\operatorname{Con} S$ is atomistic then $S$ is a tree of 0-simple semigroups. 
Proof. By Proposition 1 we observe that $S$ is semisimple and hence each principal factor is (0-)simple. Let $X=S / J . X$ is a locally finite tree. For $\alpha=J_{a}$ let $I_{\alpha}=J(a) / I(a)$. Then $I_{\alpha}^{*}=J_{a}$ and $S=\bigcup\left\{I_{\alpha}^{*}: \alpha \in X\right\}$. For $\alpha \succ$ $\alpha^{+}$let $f_{\alpha}$ be equal to the mapping $f: J_{a} \rightarrow J_{a^{+}}$such that a $\rho f f$ for some atom $\rho$ which was obtained in Lemma 4. Let $a, b \in S, a \in I_{\alpha}^{*}=J_{a}$ and $b \in I_{\beta}^{*}=J_{b}$ and let $\gamma=J_{a b}$. Let $f_{\alpha, \gamma}$ and $f_{\beta, \gamma}$ be defined according to the rules of the construction. Then by Lemma 5 we have that $\left(a f_{\alpha, \gamma}\right)\left(b f_{\beta, \gamma}\right)=$ $a b \in I_{\gamma}^{*}$. Therefore $D(a, b)$ is not empty. Also, $\gamma=J_{a b}$ is the greatest element of $D(a, b)$. To see this suppose that $d=\left(a f_{\alpha, \delta}\right)\left(b f_{\beta, \delta}\right) \in I_{\delta}^{*}$ for some $\delta \geq \gamma$. Then $a b \in J(d)$ and so again by Lemma 5 we obtain that $a b=\left(a f_{\alpha, \delta}\right)\left(b f_{\beta, \delta}\right)$ which implies that $\gamma=\delta$.

\section{Weakly reductive semigroups}

We now restrict our investigations to the case when $S$ is weakly reductive. A weakly reductive semigroup $S$ cannot be an inflation of a semigroup $T \neq$ $S$. Therefore, if Con $S$ is atomistic then weak reductivity of $S$ implies global idempotency and thus we may assume that $S=\left(X ; I_{\alpha}, f_{\alpha, \beta}\right)$, a tree of 0 -simple semigroups. In the next statements we assume that $\operatorname{Con} S$ is atomistic and $S$ is weakly reductive. Lemma 7 is straightforward to prove.

Lemma 7. Let $S=\left(X ; I_{\alpha}, f_{\alpha, \beta}\right)$. Then $S$ is weakly reductive if and only if each principal ideal of $S$ is weakly reductive.

Definition. Let $\alpha \in X$ and $x, y \in I_{\alpha}^{*}$. We define a relation $\tau_{\alpha}$ by

$$
x \tau_{\alpha} y \Leftrightarrow\left(u x v \in I_{\alpha}^{*} \Leftrightarrow u y v \in I_{\alpha}^{*} \forall u, v \in I_{\alpha}^{*}\right) .
$$

Then $\tau_{\alpha} \cup\left\{\left(0_{\alpha}, 0_{\alpha}\right)\right\}$ is the greatest congruence on $I_{\alpha}$ which saturates $I_{\alpha}^{*}$, that is, in particular, the greatest nonuniversal congruence on $I_{\alpha}$.

Lemma 8. Let $\alpha \in X^{*}$. Then the restriction of $f_{\alpha}$ to an arbitrary $\tau_{\alpha}$-class is injective.

Proof. Let $\rho_{\alpha}$ denote the greatest congruence on $S$ which saturates $I_{\alpha}^{*}$, in particular,

$$
x \rho_{\alpha} y \Leftrightarrow\left(u x v \in I_{\alpha}^{*} \Leftrightarrow u y v \in I_{\alpha}^{*} \forall u, v \in S^{1}\right) .
$$

We will prove that $\rho_{\alpha} \mid I_{\alpha}^{*}=\tau_{\alpha}$. Obviously we have that $\rho_{\alpha} \mid I_{\alpha}^{*} \subseteq \tau_{\alpha}$. Suppose that $x \tau_{\alpha} y$ but $(x, y) \notin \rho_{\alpha}$. We may assume there exist $u, v \in S^{1}$ such that $u x v \in I_{\alpha}^{*}$ and $u y v \notin I_{\alpha}^{*}$ and $\{u, v\} \cap S \neq \varnothing$. Then $J_{u}, J_{v} \geq J_{x}$ and 
so $u x v=(u f) x(v g)$ and $u y v=(u f) y(v g)$ such that $u f, v g \in\left(I_{\alpha}^{*}\right)^{1}$ and $\{u f, v g\} \cap I_{\alpha}^{*} \neq \varnothing$. If $\{u f, v g\} \subseteq I_{\alpha}^{*}$ then the proof is finished. If not then we may suppose that $u f \in I_{\alpha}^{*}$ and $v \notin S$. Since $I_{\alpha}$ is 0 -simple there exists $w \in I_{\alpha}^{*}$ such that $(u f) x w \in I_{\alpha}^{*}$. Then $(u f) y w \notin I_{\alpha}^{*}$ since $(u f) y \notin I_{\alpha}^{*}$. This again is a contradiction to $x \tau_{\alpha} y$.

Now suppose that $x f_{\alpha}=y f_{\alpha}$ for $x, y$ such that $x \tau_{\alpha} y$. Then $x \rho x f_{\alpha}=$ $y f_{\alpha} \rho y$ for some $\rho \in$ At $S$. $\rho$ does not saturate $I_{\alpha}^{*}$ thus $\rho \cap \rho_{\alpha} \neq \rho$ which implies that $\rho \cap \rho_{\alpha}=\varepsilon$. Therefore $x=y$.

LEMMA 9. Let $\alpha \in X^{*}$. Then the restriction of $f_{\alpha}$ to an arbitrary $\tau_{\alpha}$-class is constant.

Proof. Let $x, y \in I_{\alpha}^{*}$ with $x \tau_{\alpha} y$. The congruence $\rho_{\alpha}$ as defined in Lemma 8 , is a supremum of atoms. So $x=a_{0} \rho_{1} a_{1} \cdots \rho_{n} a_{n}=y$ for certain elements $a_{i}$ and atoms $\rho_{i} \subseteq \rho_{\alpha}$. Since $\rho_{\alpha}$ saturates $I_{\alpha}^{*}$, we observe that $a_{i} \in I_{\alpha}^{*}$ for all $i$. Let $z \in I_{\gamma}^{*}$ for some $\gamma<\alpha$. We have that $\rho_{i} \mid I_{\delta}^{*}=\varepsilon$ for all $\delta<\alpha$ since $\rho_{i}$ is an atom. Hence $a_{i} z=a_{i+1} z$ and $z a_{i}=z a_{i+1}$ for all $i$. Multiplying the sequence $x=a_{0} \rho_{1} a_{1} \cdots \rho_{n} a_{n}=y$ by $z$ on the left and right, respectively, we obtain that $\left(x f_{\alpha}\right) z=x z=y z=\left(y f_{\alpha}\right) z$ and $z\left(x f_{\alpha}\right)=z x=z y=z\left(y f_{\alpha}\right)$, respectively. Weak reductivity of the semigroup $I=\bigcup\left(I_{\gamma}^{*}: \gamma<\alpha\right)$ then implies that $x f_{\alpha}=y f_{\alpha}$.

Proposition 2. If $\alpha$ is not minimal in $X$ then $I_{\alpha}$ is congruence free.

Proof. $\tau_{\alpha}$ is the identical relation. Therefore, by Result $1, I_{\alpha}$ is congruence free.

Lemma 10. Let $S=\left(X ; I_{\alpha}, f_{\alpha, \beta}\right)$ be a tree of 0-simple semigroups. Let $\alpha \geq \beta \geq \gamma \geq \delta \in X$ and $x \rho y$ for some $x \in I_{\alpha}^{*}, y \in I_{\delta}^{*}$ and $\rho \in \operatorname{Con} S$. Then $z f_{\beta, \gamma} \rho z$ for all $z \in I_{\beta}^{*}$.

Proof. See [1, Lemma 9].

Using the following definition, the mapping $f_{\alpha}$ may be regarded as a binary relation on $S$ :

$$
x f_{\alpha} y \Leftrightarrow x \in I_{\alpha}^{*} \text { and } x f_{\alpha}=y .
$$

LEMMA 11. Let $S=\left(X ; I_{\alpha}, f_{\alpha, \beta}\right)$ be a tree of congruence free semigroups. Then $\rho \in \operatorname{Con} S$ is an atom if and only if $\rho=\left(f_{\alpha} \cup \varepsilon_{S}\right) \circ\left(f_{\alpha}^{-1} \cup \varepsilon_{S}\right)$ for some $\alpha \in X^{*}$. 
Proof. Let $\xi=\left(f_{\alpha} \cup \varepsilon_{S}\right) \circ\left(f_{\alpha}^{-1} \cup \varepsilon_{S}\right)$ for some $\alpha \in X$. Then for $u \neq v$ we have $u \xi v$ if and only if $u=v f_{\alpha}, v=u f_{\alpha}$ or $u f_{\alpha}=v f_{\alpha}$. It can be seen easily that $\xi$ is a congruence. Let $\eta$, where $\varepsilon \neq \eta \subseteq \xi$, be a congruence. Then $u \eta v$ for some $u \neq v$. If $u=v f_{\alpha}$ then $z \eta z f_{\alpha}$ for all $z \in I_{\alpha}^{*}$ by Lemma 10 and so $\eta=\xi$. If $u f_{\alpha}=v f_{\alpha}$ and $u \neq v$ then there exist $x, y \in I_{\alpha}^{*}$ such that $x u y \in I_{\alpha}^{*}$ and $x v y \notin I_{\alpha}^{*}$, or conversely. Again by Lemma 10, we obtain that $\eta=\xi$. Conversely, let $\rho$ be an arbitrary congruence and $x \rho y$ for $x \neq y$ where $x \in I_{\alpha}^{*}, y \in I_{\beta}^{*}$. If $\alpha \neq \beta$ then we assume that $\alpha \beta<\alpha$. It is easy to see that $z f_{\alpha, \alpha \beta} \rho z$ for all $z \in I_{\alpha}^{*}$ and thus $\left(f_{\alpha} \cup \varepsilon_{S}\right) \circ\left(f_{\alpha}^{-1} \cup \varepsilon_{S}\right) \subseteq \rho$. If $\alpha=\beta$ and $x \neq y$ then by the same argument as in the first half of the proof we obtain that $\left(f_{\alpha} \cup \varepsilon_{S}\right) \circ\left(f_{\alpha}^{-1} \cup \varepsilon_{S}\right) \subseteq \rho$.

LEMMA 12. Let $S=\left(X ; I_{\alpha}, f_{\alpha, \beta}\right)$ be a tree of congruence free semigroups where $X$ has no least element. If Con $S$ is atomistic then for $x, y \in I_{\alpha}^{*}$ there exists $\gamma \leq \alpha$ such that $x f_{\alpha, \gamma}=y f_{\alpha, \gamma}$.

Proof. Let $x, y \in I_{\alpha}^{*}$ and $x=x_{0} \rho_{1} x_{1} \cdots \rho_{n} x_{n}=y$ for some atoms $\rho_{i}$ such that all $x_{i} \in J(x)$. For $x \neq y$ let $g(x, y)=n$ denote the smallest length of such a sequence. We prove the assertion by induction on $g(x, y)$. If $g(x, y)=1$ then by Lemma $11, x f_{\alpha}=y f_{\alpha}$. Let $g(x, y)=n>1$ and suppose that the assertion is true whenever $g(u, v)<n$. Let $\alpha_{i}$ be defined by $x_{i} \in I_{\alpha_{i}}^{*}$. Then $\alpha_{i} \leq \alpha$ for all $i$ since $x_{i} \in J(x)$. If $\alpha_{i}=\alpha$ for all $i$ then $x_{0} f_{\alpha}=x_{1} f_{\alpha}=\cdots=x_{n} f_{\alpha}=y f_{\alpha}$ which is a contradiction to $g(x, y)>1$. Let $j$ be the first index such that $\alpha_{j}<\alpha$. Then $x_{0} f_{\alpha}=x_{1} f_{\alpha}=\cdots=x_{j-1} f_{\alpha}=x_{j}$. Therefore $j=1: x_{1}=x_{0} f_{\alpha}=x f_{\alpha}$. By the same argument we obtain that $x_{n-1}=x_{n} f_{\alpha}=y f_{\alpha}$. Now there are two alternatives: (i) $x_{i} \in J\left(x_{1}\right)=J\left(x_{n-1}\right)$ for all $1 \leq i \leq n-1$ and (ii) there exists $i, 1 \leq i \leq n-1$, such that $x_{i} \in$ $I_{\alpha}^{*}=J_{x_{0}}=J_{x_{n}}$. Since all $x_{i} \in J(x)$ only these two cases are possible. For the first case we have that $g\left(x_{1}, x_{n-1}\right)<n$ and so $x_{1} f_{\alpha^{+}, \gamma}=x_{n-1} f_{\alpha^{+}, \gamma}$ for some $\gamma \leq \alpha^{+}$. Then $x f_{\alpha, \gamma}=x_{1} f_{\alpha^{+}, \gamma}=x_{n-1} f_{\alpha^{+}, \gamma}=y f_{\alpha, \gamma}$. In the second case we have $g\left(x, x_{i}\right)<n$ and $g\left(y, x_{i}\right)<n$ and therefore $x f_{\alpha, \gamma}=x_{i} f_{\alpha, \gamma}=y f_{\alpha, \gamma}$ for some $\gamma<\alpha$.

Of course the condition of Lemma 12 is equivalent to the condition: for any $a \in I_{\alpha}^{*}$ and $b \in I_{\beta}^{*}$ there exists $\gamma \leq \alpha, \beta$ such that $a f_{\alpha, \gamma}=b f_{\beta, \gamma}$.

Using the following known lemmas, we thus have obtained a characterization of weakly reductive semigroups with atomistic congruence lattices.

Notation. For an arbitrary set $X$, let $P(X)$ be the lattice of all subsets of $X$.

Lemma 13. Let $X$ be a locally finite tree. Then $\operatorname{Con} X \cong \mathcal{P}\left(X^{*}\right)$. 
Proof. See [3, Lemma 3].

LEMMA 14. Let $S=\left(X ; I_{\alpha}, f_{\alpha, \beta}\right)$ be a tree of congruence free semigroups $I_{\alpha}$ such that for all $a \in I_{\alpha}^{*}$ and $b \in I_{\beta}^{*}$ there exists $\gamma$ satisfying $a f_{\alpha, \gamma}=$ $b f_{\beta, \gamma}$. Then $\operatorname{Con} S \cong \operatorname{Con} X$.

Proof. The lemma is a consequence of the proof of [2, Theorem 8$]$.

LEMMA 15. Let $S=\left(X ; I_{\alpha}, f_{\alpha, \beta}\right)$ be a tree of 0 -simple semigroups where $I_{\alpha}$ is congruence free for all $\alpha \in X^{*}$ and $X$ has a least element $\mu$. Then Con $S \cong \operatorname{Con} X \times \operatorname{Con} I_{\mu}^{*}$.

Proof. The lemma is a consequence of the proof of [2, Theorem 8].

Using this and the fact that a product of two lattices is atomistic if and only if each factor is atomistic, we can formulate

THEOREM 2. Let $S$ be a weakly reductive semigroup. Then $\operatorname{Con} S$ is atomistic if and only if $S$ is isomorphic to one of the following:

(i) a simple semigroup I such that Con $I$ is atomistic;

(ii) a tree of congruence free semigroups $\left(X ; I_{\alpha}, f_{\alpha, \beta}\right)$ such that for each $x \in I_{\alpha}^{*}, y \in I_{\beta}^{*}$ there exists $\gamma \leq \alpha, \beta$ satisfying $x f_{\alpha, \gamma}=y f_{\beta, \gamma}$;

(iii) a tree of 0 -simple semigroups $\left(X ; I_{\alpha}, f_{\alpha, \beta}\right)$ where $X$ has a least element $\mu$ such that $I_{\mu}^{*}$ is a semigroup of type (i) and $S / I_{\mu}^{*}$ is a semigroup of type (ii).

\section{Applications}

In order to study special classes of semigroups we first need a result for groups.

Proposition 3. A group has an atomistic congruence lattice if and only if it is a direct sum of simple groups.

Proof. For a group we may identify congruences and normal subgroups.

NECESSITY. Suppose that the group $G$ has an atomistic congruence lattice. Let $\left\{N_{i}: i \in I\right\}$ be the set of all atoms of the lattice of normal subgroups of $G$. Then $G=\bigvee\left(N_{i}: i \in I\right)$. Let $A$ be defined by

$$
\mathcal{A}=\left\{K \subseteq I: \forall i \in K: N_{i} \cap \bigvee\left(N_{j}: j \in K \backslash\{i\}\right)=\{1\}\right\}
$$


Then $A$ is not empty. Let $C \subseteq A$ be a chain and $J=\cup C$. If $J \notin A$ then there exists $j \in J$ such that $N_{j} \subseteq \bigvee\left(N_{i}: i \in J, i \neq j\right)$. Let $n \in N_{j}$, $n \neq 1$. Then there exist $i_{1}, \ldots, i_{k} \in J \backslash\{j\}$ such that $n \in N_{i_{1}} \vee \cdots \vee N_{i_{k}}$. Then $N_{j} \cap\left(N_{i_{1}} \vee \cdots \vee N_{i_{k}}\right) \neq\{1\}$ and so $N_{j} \subseteq N_{i_{1}} \vee \cdots \vee N_{i_{k}}$ because $N_{j}$ is an atom. This is a contradiction to the definition of $A$ because there exists $C \in C$ which contains the indices $i_{h}$ as well as $j$. Therefore $J \in A$. Now by Zorn's Lemma there exists a maximal element in $A$, to be denoted by $K$. If $K=I$ then we obviously have that $G=\sum\left(N_{i}: i \in I\right)$. Now suppose that $K \neq I$. Let $j \in I \backslash K$. If $N_{j}$ is not contained in $\bigvee\left(N_{k}: k \in\right.$ $K)$ then there exists $i \in K$ such that $N_{i} \subseteq \bigvee\left(N_{k}: k \in K, k \neq i\right) \vee N_{j}$ because $K \cup\{j\} \notin \mathcal{A}$. Let $N=\bigvee\left(N_{k}: k \in K, k \neq i\right)$. Then we obtain that $\left\{\{1\}, N_{j}, N, N \vee N_{i}, N \vee N_{j}\right\}$ forms a non-modular sublattice of the lattice of all normal subgroups of $G$, a contradiction. Therefore, $N_{j} \subseteq \bigvee\left(N_{k}: k \in K\right)$ which implies that $G=\sum\left(N_{k}: k \in K\right)$. Then each normal subgroup of some $N_{i}$ is a normal subgroup of $G$ and therefore all $N_{i}$ are simple groups.

SUFFICIENCY. Let $G=\sum G_{i}$ be a direct sum of simple groups $G_{i}$. Let $N$ be a normal subgroup of $G$ and $n \in N$. Then $n=a_{1} \cdots a_{k} b$ where the element $a_{i}$ belongs to some non-commutative group $G_{i}$ and $b$ belongs to the centre of $G$. To each $a_{i}$ there exists $c_{i} \in G_{i}$ such that $a_{i} c_{i} \neq c_{i} a_{i}$. Then $n c_{i} n^{-1} c_{i}^{-1}=a_{i} c_{i} a_{i}^{-1} c_{i}^{-1} \in N \cap G_{i}$ and $a_{i} c_{i} a_{i}^{-1} c_{i}^{-1} \neq 1$. Since $G_{i}$ is simple $G_{i} \subseteq N$. In particular, $a_{i} \in N$ for all $i$ and therefore $b \in N$. The order of $b$ is square free: $o(b)=p_{1} \cdots p_{s}$ for some distinct primes $p_{j}$. Let $q_{j}=p_{1} \cdots p_{s} / p_{j}$. Then $\left\langle b^{q_{j}}\right\rangle \cong \mathbb{Z}_{p_{j}}$ and $\left\langle b^{q_{j}}\right\rangle \subseteq N$. The groups $G_{i}$ and $\left\langle b^{q_{j}}\right\rangle$ are atoms in the lattice of all normal subgroups of $G$. Then $n \in G_{1} \cdots G_{k}\left\langle b^{q_{1}}\right\rangle \cdots\left\langle b^{q_{s}}\right\rangle \subseteq N$ implies that $N$ is the supremum of the atoms it contains.

\subsection{Commutative semigroups.}

We first treat the globally idempotent case. A commutative semigroup is 0 -simple if and only if it is a commutative group with a zero adjoined. Such a semigroup is congruence free if and only if its non-zero part consists of only one (idempotent) element. So $S=\left(X ; I_{\alpha}, f_{\alpha, \beta}\right)$, the tree of congruence free semigroups, degenerates to the locally finite tree $X$. Furthermore, a commutative group has an atomistic lattice of subgroups if and only if it a direct sum of cyclic groups $\mathbb{Z}_{p}$ of prime order. So for the globally idempotent case we have exactly the three cases: (i) a direct sum of cyclic groups $\mathbb{Z}_{p}$ of prime order, (ii) a locally finite tree, (iii) an ideal extension of a semigroup $G$ as (i) by a semigroup $X$ as (ii) with zero.

For the general case we need the following proposition. 
Proposition 4. Let $T$ be an inflation of a semigroup $S$ such that all homomorphic images of $S$ are weakly reductive. The Con $T$ is atomistic if and only if $\operatorname{Con} S$ is atomistic and the inflation function $f$ is trivial, that is $|(T \backslash S) f|=1$.

Proof. Suppose that Con $T$ is atomistic. For each congruence $\rho$ on $S$, $\rho \cup \varepsilon_{T}$ is a congruence on $T$. Therefore, if $\operatorname{Con} T$ is atomistic the same holds for Con $S$. Let $\rho$ be defined by $x \rho y$ if and only if $x, y \in S$ or $x, y \in T \backslash S$. Let $x, y \in T \backslash S$ and $x=x_{0} \rho_{1} x_{1} \cdots \rho_{n} x_{n}=y$ for some atoms $\rho_{i} \subseteq \rho$. Since the $\rho_{i}$ 's are atoms we have that $\rho_{i} \mid S=\varepsilon$. Therefore, $(x f) z=x z=y z=(y f) z$ and $z(x f)=z x=z y=z(y f)$ for all $z \in S$. Weak reductivity of $S$ then implies that $x f=y f$. Conversely, let $T$ be an inflation of a semigroup $S$ such that all homomorphic images of $S$ are weakly reductive, suppose that $\operatorname{Con} S$ is atomistic and $|(T \backslash S) f|=1$ where $f$ stands for the inflation function. Let $a \in S$ denote the element of $S$ which defines the multiplication of the inflation, that is $x f=a$ for all $x \in T \backslash S$. Let $x \in T \backslash S, b \in S$ and suppose that $x \rho b$ for some $\rho \in \operatorname{Con} T$. We obtain that $x z=a z \rho b z$ and $z x=z a \rho z b$ for all $z \in S$. Since $S /(\rho \mid S)$ is weakly reductive we have $a \rho b$. Now we may apply Lemma 11 in [2] which proves that under this condition the mapping $\rho \rightarrow(\rho|S, \rho| T \backslash S \cup\{a\})$ is an isomorphism between $\operatorname{Con} T$ and $\operatorname{Con} S \times \operatorname{Eq} T \backslash S \cup\{a\}$.

Summarizing these observations we may formulate

THEOREM 3. A commutative semigroup $S$ has an atomistic congruence lattice if and only if $S$ is isomorphic to one of the following:

(i) a direct sum of cyclic groups $\mathbb{Z}_{p}$ of prime order;

(ii) a locally finite tree;

(iii) an ideal extension of a semigroup of type (i) by a semigroup of type (ii) with zero;

(iv) an inflation of a semigroup of type (i), (ii) or (iii) with a trivial inflation function.

We observe that for commutative semigroups $S$ the conditions "Con $S$ is atomistic" and "Con $S$ is relatively complemented" are equivalent (see [2, Corollary 14]).

\subsection{Finite semigroups.}

Again we first treat the globally idempotent case. Put $S=\left(X ; I_{\alpha}, f_{\alpha, \beta}\right)$, a tree of 0 -simple semigroups. Finiteness implies that all $I_{\alpha}$ are completely 0 -simple. If $\alpha$ is not minimal in $X$ then $I_{\alpha}$ is congruence free and therefore $I_{\alpha} \cong \mathcal{M}^{0}\left(I_{\alpha}, \Lambda_{\alpha}, P_{\alpha}\right)$ where $P_{\alpha}$ is a $\Lambda_{\alpha} \times I_{\alpha}$-matrix of zeros and ones such that each row and each column contain a one and no two rows and no two columns are identical (see [11] or [6]). $X$ has a least element $\mu$ and $I_{\mu}^{*}$ is 
a completely simple semigroup. Therefore, $I_{\mu}^{*} \cong \mathcal{M}(I, G, \Lambda, P)$. Suppose that $\operatorname{Con} I_{\mu}^{*}$ is atomistic. $\operatorname{Con} I_{\mu}^{*}$ is isomorphic to $\operatorname{Ad}=\operatorname{Ad}(I, G, \Lambda, P)$, a sublattice of $\mathrm{Eq} I \times \operatorname{Nor} G \times \operatorname{Eq} \Lambda$, the lattice of admissible triples (see [6]) (Nor $G$ denotes the lattice of all normal subgroups of $G$ ). An element $(\xi, N, \eta) \in \mathrm{Eq} I \times \operatorname{Nor} G \times \mathrm{Eq} \Lambda$ is admissible if

$$
i \xi j \Rightarrow p_{\lambda i} p_{\mu i}^{-1} p_{\mu j} p_{\lambda j}^{-1} \in N \quad \forall \lambda, \mu \in \Lambda
$$

and

$$
\lambda \eta \mu \Rightarrow p_{\lambda i} p_{\mu i}^{-1} p_{\mu j} p_{\lambda j}^{-1} \in N \quad \forall i, j \in I .
$$

All elements of the form $(\varepsilon, N, \varepsilon)$ are admissible. Furthermore if $(\xi, N, \eta)$ is admissible and $\zeta \subseteq \xi$ then $(\zeta, N, \varepsilon)$ is also admissible. $(\omega, G, \omega)$ is admissible and hence the supremum of admissible atoms. If $(\zeta, N, \varepsilon)$ is an atom in $\operatorname{Ad}(I, G, \Lambda, P)$ and $\zeta \neq \varepsilon$ then $N=\{1\}$ and $\zeta$ is an atom in Eq $I$. Let $i, j \in I$ and $\lambda \in \Lambda$. There exist atoms $\rho_{1}, \ldots, \rho_{n}$ in Ad such that

$$
(i, 1, \lambda) \rho_{1} \cdots \rho_{n}(j, 1, \lambda) .
$$

Each $\rho_{k}$ whose first entry is a proper equivalence commutes with each $\rho_{k}$ whose first entry is the identity. Therefore in (1) we may omit the latter ones. Thus for each $i, j \in I$ there exists $\xi \in \mathrm{Eq} I$ such that $i \xi j$ and $(\xi,\{1\}, \varepsilon)$ is admissible and therefore $(\omega,\{1\}, \varepsilon)$ is admissible. The same holds for $(\varepsilon,\{1\}, \omega)$ and thus $(\omega,\{1\}, \omega)$ is also admissible. We have thus obtained that all triples are admissible and then $I_{\mu}^{*} \cong I \times G \times \Lambda$, a rectangular group (see [8]). Since each partition lattice is atomistic $\operatorname{Con} I_{\mu}^{*}$ is atomistic if and only if Nor $G$ is atomistic, that is if and only if $G$ is a direct sum of simple groups. If $S$ is a tree of congruence free semigroups then finiteness implies that $X$ has a least element $\mu$. Then $\left|I_{\mu}^{*}\right|=1$ and therefore $x f_{\alpha, \mu}=y f_{\beta, \mu}$ for arbitrary $x \in I_{\alpha}^{*}$ and $y \in I_{\beta}^{*}$. Since Proposition 4 here also applies, we can formulate

THEOREM 4. Let $S$ be a finite semigroup. Then $\operatorname{Con} S$ is atomistic if and only if $S$ is isomorphic to one of the following.

(i) a rectangular group $I \times G \times \Lambda$ such that $G$ is a direct sum of simple groups;

(ii) a tree of congruence free semigroups;

(iii) a tree of 0-simple semigroups $\left(X ; I_{\alpha}, f_{\alpha, \beta}\right)$ such that $I_{\mu}^{*}$ is a semigroup of type (i) (where $\mu$ denotes the least element of $X$ ) and $S / I_{\mu}^{*}$ is a semigroup of type (ii);

(iv) an inflation of a semigroup of type (i), (ii) or (iii) with a trivial inflation function. 
In [4, Theorems 3.11 and 3.14] the partial homomorphisms between nonzero parts of completely 0 -simple semigroups are described.

Completely semisimple semigroups can be treated in the same way as the globally idempotent case of finite semigroups, omitting the finiteness conditions. Here it may happen that the locally finite tree $X$ of $S=\left(X ; I_{\alpha}, f_{\alpha, \beta}\right)$ has no least element and so in (ii) the condition "for $x \in I_{\alpha}^{*}$ and $y \in I_{\beta}^{*}$ there exists $\gamma \leq \alpha, \beta$ such that $x f_{\alpha, \gamma}=y f_{\beta, \gamma}$ " must be added. An example in [1] shows that this is really necessary. In [2, Section 5] a necessary and sufficient condition for this property is given. The present section is closely related to [2, Section 5]. Again for finite and completely semisimple semigroups the properties "Con $S$ is atomistic" and " $\operatorname{Con} S$ is relatively complemented" are equivalent.

\section{References}

[1] K. Auinger, 'Semigroups with complemented congruence lattices', Algebra Universalis 22 (1986), 192-204.

[2] K. Auinger, 'Semigroups with Boolean congruence lattices', Monatsh. Math. 104 (1987), $1-15$.

[3] K. Auinger, 'Atomistic congruence lattices of semilattices', Semigroup Forum, to appear.

[4] A. H. Clifford and G. B. Preston, 'The algebraic theory of semigroups', Vol. 1, Amer. Math. Soc. Math. Surveys, No. 7, (Providence, R.I., 1961).

[5] L. M. Gluskin, 'Simple semigroups with zero', Dokl. Akad. Nauk SSSR 103 (1955), 5-8. (Russian)

[6] J. M. Howie, An introduction to semigroup theory, (Academic Press, New York, 1976).

[7] G. Lallement and M. Petrich, 'Structure d'une classe de demi-groupes reguliers, J. Math. Pures Appl. 48 (1969), 345-397.

[8] M. Petrich, Introduction to semigroups, (Merrill, Columbus, 1973).

[9] M. Petrich, 'Structure of regular semigroups', Cahiers Math. Montpellier 11, (Université du Languedoc, Montpellier, 1977).

[10] B. M. Schein, 'Homomorphisms and subdirect decompositions of semigroups', Pacific J. Math. 17 (1966), 529-547.

[11] T. Tamura, 'Indecomposable completely simple semigroups except groups', Osaka Math. J. 8 (1956), 35-42.

[12] E. J. Tully, Jr., 'Semigroups in which each ideal is a retract', J. Austral. Math. Soc. 9 (1969), 239-245.

Institut für Mathematik

Universität Wien

A-1090 Wien

Austria 